\title{
The Ichikado CT score as a prognostic tool for coronavirus disease 2019 pneumonia: a retrospective cohort study
}

\author{
Alan Araiza ${ }^{1,2}$, Melanie Duran ${ }^{1,3}$, Cesar Patiño ${ }^{1,4}$, Paul E. Marik ${ }^{1}$ and Joseph Varon ${ }^{1,5^{*}}$
}

\begin{abstract}
Background: The relationship between computed tomography (CT) and prognosis of patients with COVID-19 pneumonia remains unclear. We hypothesized that the Ichikado CT score, obtained in the first $24 \mathrm{~h}$ of hospital admission, is an independent predictor for all-cause mortality during hospitalization in patients with COVID-19 pneumonia.

Methods: Single-center retrospective cohort study of patients with confirmed COVID-19 pneumonia admitted at our institution between March 20th, 2020 and October 31st, 2020. Patients were enrolled if, within 24 h of admission, a chest CT scan, an arterial blood gas, a complete blood count, and a basic metabolic panel were performed. Two independent radiologists, who were blinded to clinical data, retrospectively evaluated the chest CT scans following a previously described qualitative and quantitative $C T$ scoring system. The primary outcome was all-cause in-hospital mortality or survival to hospital discharge. Secondary outcomes were new requirements for invasive mechanical ventilation and hospital length of stay. Cox regression models were used to test the association between potential independent predictors and all-cause mortality.
\end{abstract}

Results: Two hundred thirty-five patients, 197 survivors and 38 nonsurvivors, were studied. The median Ichikado CT score for nonsurvivors was significantly higher than survivors $(P<0.001)$. An Ichikado $C T$ score of more than 172 enabled prediction of mortality, with a sensitivity of $84.2 \%$ and a specificity of $79.7 \%$. Multivariate analysis identified Ichikado CT score (HR, 7.772; 95\% Cl, 3.164-19.095; $P<0.001)$, together with age (HR, 1.030; 95\% Cl, 1.030-1.060; $P=0.043)$, as independent predictors of all-cause in-hospital mortality.

Conclusions: Ichikado CT score is an independent predictor of both requiring invasive mechanical ventilation and all-cause mortality in patients hospitalized with COVID-19 pneumonia. Further prospective evaluation is necessary to confirm these findings.

Trial registration: The WCG institutional review board approved this retrospective study and patient consent was waived due to its non-interventional nature (Identifier: 20210799).

Keywords: Coronavirus disease 2019, COVID-19, CT score, Ichikado CT score, COVID-19 prognostic factor, Tomography

*Correspondence: jvaron@roamer.net

${ }^{5}$ University of Texas Health Science Center at Houston, Houston, TX, USA

Full list of author information is available at the end of the article

\section{Background}

In late December 2019, a cluster of pneumonia cases of unknown origin was reported in Wuhan, Hubei Province, China [1]. Days later, the Chinese health authorities confirmed that coronavirus disease 2019 (COVID-19) was caused by severe acute respiratory coronavirus 2 original author(s) and the source, provide a link to the Creative Commons licence, and indicate if changes were made. The images or other third party material in this article are included in the article's Creative Commons licence, unless indicated otherwise in a credit line to the material. If material is not included in the article's Creative Commons licence and your intended use is not permitted by statutory regulation or exceeds the permitted use, you will need to obtain permission directly from the copyright holder. To view a copy of this licence, visit http://creativecommons.org/licenses/by/4.0/. The Creative Commons Public Domain Dedication waiver (http://creativeco mmons.org/publicdomain/zero/1.0/) applies to the data made available in this article, unless otherwise stated in a credit line to the data. 
(SARS-CoV-2) [2]. On March 11th 2020, the World Health Organization declared the COVID-19 outbreak a pandemic, as by then the cases of COVID-19 had spread to over 110 countries [3]. By February 14th 2021, the United States of America had a total of 27,221,607 cases of COVID-19, and 477,147 fatalities [4].

Coronavirus disease 2019 was initially thought to be a pulmonary-limited disease; however, we have learned that in moderate-to-severe cases it is accompanied by a systemic dysregulated inflammatory response and a hypercoagulable state [5-7]. The most common clinical presentation includes fever, cough, myalgias, fatigue and anosmia. While most patients will have mild symptoms and recover without medical management, some will develop dyspnea, requiring supplemental oxygen therapy, and eventually worsen to respiratory or multi-organ failure, requiring mechanical ventilation and intensive care unit (ICU) monitoring $[8,9]$. As the number of people infected by SARS-CoV-2 continues to climb, early recognition of patients at risk of deterioration may aid in the triage and medical management of COVID-19 patients [10].

Most studies have focused on inflammatory biomarkers as predictive tools [11-13]. Data regarding prognostic value of chest computed tomography $(\mathrm{CT})$ is scarce. To our knowledge, none of the chest CT scoring systems proposed for COVID-19 patients are quantitative and consider both the percentage of lung parenchyma affected and the type of infiltrate [10, 14-19]. In 2006, Ichikado et al. proposed and validated a chest $\mathrm{CT}$ scoring system for acute respiratory distress syndrome (ARDS) patients which proved to be an independent predictive factor for survival, ventilator-free days, and barotrauma incidence in mechanically ventilated patients [20].

The aim of this study was to determine the prognostic value of the Ichikado CT score upon admission for allcause mortality in patients admitted to a COVID-19 ICU or intermediate care unit (IMU). We hypothesized that the Ichikado CT score is a useful predictive tool for hospital mortality.

\section{Methods}

\section{Study population and design}

We performed a single-center retrospective cohort study of patients with COVID-19 pneumonia admitted between March $20^{\text {th }}, 2020$ and October $31^{\text {st }}, 2020$. Two hundred forty-one patients with confirmed SARS-CoV-2 infection were admitted at our institution's COVID-19 ICU and IMU. Infection was confirmed by reverse transcription polymerase chain reaction (RT-PCR), SARS Antigen Fluorescent Immunoassay (SOFIA), or IgG/IgM rapid tests, plus clinical and imaging correlation.
Patients were enrolled in our analysis if within $24 \mathrm{~h}$ from admission they underwent a chest $\mathrm{CT}$, in supine or prone position, with or without intravenous contrast, and if within the same timeframe they had an arterial blood gas (ABG), a complete blood count, and a basic metabolic panel. Patients were excluded if they were younger than 18 years of age, they had a history of chronic interstitial lung disease or had missing demographic or outcome data. Considering the total patient population at our institution's COVID-19 units, we calculated requiring a sample size of 149 patients to achieve a 95\% confidence level.

The WCG institutional review board approved this retrospective study and patient consent was waived due to its non-interventional nature (Identifier: 20210799). This study was conducted in accordance with the amended Declaration of Helsinki.

\section{Data collection}

Data were abstracted from both physical and digital hospital archives between February 24th, 2021 and February 25th, 2021. Two authors performed data entry to an electronic spreadsheet where information was doublechecked. The demographic data included was age, gender, and race (African-American, Caucasian, Hispanic, Other). The clinical data included comorbidities like hypertension, diabetes mellitus, chronic obstructive pulmonary disease (COPD), chronic kidney disease (CKD) or end-stage renal disease (ESRD), the days since symptom onset until hospital admission, arterial partial pressure of oxygen $\left(\mathrm{PaO}_{2}\right)$, arterial partial pressure of carbon dioxide $\left(\mathrm{PaCO}_{2}\right)$, fraction of inspired oxygen $\left(\mathrm{FiO}_{2}\right)$ at the time of ABG, arterial partial pressure of oxygen-to-fraction of inspired oxygen ratio $\left(\mathrm{PaO}_{2}: \mathrm{FiO}_{2}\right)$, alveolar-arterial (A-a) gradient, Sequential Organ Failure Assessment (SOFA) score, absolute neutrophil count, absolute lymphocyte count, absolute neutrophil-to-lymphocyte ratio (NLR), Ichikado CT score, oxygen delivery device used at the time of CT scan and bloodwork obtention, medications utilized during hospitalization (corticosteroids, ascorbic acid, thiamine, anticoagulation, tocilizumab or remdesivir), prone positioning, renal replacement therapy (RRT), vasopressor use, acute kidney injury (AKI) during hospitalization, hospital length of stay (LoS) and new requirements for invasive mechanical ventilation. Fraction of inspired oxygen was estimated according to the oxygen delivery device used at the time of ABG. If patients had multiple laboratory measurements within the $24 \mathrm{~h}$ time frame, the worst clinical value was considered for this study.

Patients who underwent mechanical ventilation, before or after CT scan obtention, followed the low PEEP/high $\mathrm{FiO}_{2}$ ARDSnet protocol for lung protective ventilation, 
with additional attention to maintain a plateau pressure below $27 \mathrm{cmH}_{2} \mathrm{O}$ and a driving pressure below $15 \mathrm{cmH}_{2} \mathrm{O}$ [21]. Prone positioning was done in both intubated and non-intubated patients, following the institutional protocol of $16 \mathrm{~h}$ of prone position and $8 \mathrm{~h}$ of supine position.

\section{CT scan examination}

Two independent radiologists with more than 10 years of experience of chest CT scan interpretation, who were blinded to clinical data, evaluated the chest $\mathrm{CT}$ scans included in our analysis. The observers followed a previously described qualitative and quantitative CT scoring system [20]. The visual scoring focused on the presence and extent of areas with normal lung parenchyma attenuation, ground-glass opacity (GGO), airspace consolidation, traction bronchiectasis or bronchiolectasis, and crazy-paving. Ground-glass attenuation was considered present if there was an area of opacification without obscuring the pulmonary vascular markings; airspace consolidation was defined as opacifications obscuring underlying vascular markings; traction bronchiectasis or bronchiolectasis were recognized as areas of irregularly dilated bronchi or bronchioles, respectively; presence of cystic air spaces with well-defined walls were defined as crazy-paving.

The previously described findings were graded on a scale of 1-6: 1, normal attenuation; 2, GGO; 3 , airspace consolidation; 4, GGO with traction bronchiectasis or bronchiolectasis; 5 , airspace consolidation with traction bronchiectasis or bronchiolectasis; 6, crazy-paving. Each attenuation was assessed in three (upper, middle, and lower) zones of each lung. The upper zone was defined as the lung parenchyma above the carina, the middle zone as the area between the carina and the pulmonary vein, and the lower zone as the infrapulmonary vein area (Fig. 1). The extent of each aberration was visually estimated to the nearest $10 \%$ of the lung parenchyma affected in each zone. The score for each zone was calculated by multiplying the percentage area by its respective point value (the score of 1 to 6). The six zones scores were averaged to determine an overall CT score for each patient (Fig. 2). The visual scoring of both radiologists was averaged, yielding a final score which was utilized in our analysis.

\section{Outcomes}

The primary endpoint was all-cause mortality during hospitalization or survival to hospital discharge. Additionally, secondary endpoints were duration of hospital LoS, and new requirements for invasive mechanical ventilation after the time of $\mathrm{CT}$ examination.

\section{Statistical analysis}

Continuous variables were expressed as mean \pm standard deviation (SD) or median with interquartile range (IQR), depending on normality of distribution, and analyzed using a non-paired Student's $t$ test or Mann-Whitney U test, as appropriate. Categorical variables were expressed as frequencies and percentages, and compared using a Chi-squared test. Interobserver variability among radiologists, in chest CT scoring, was assessed with intraclass correlation coefficient. Correlations between Ichikado CT score and clinical data were determined using Spearman's rank correlation coefficient.

Univariable and multivariable Cox regression models were used to test the association between potential independent predictive factors and all-cause mortality during COVID-19 hospitalization. Variables included

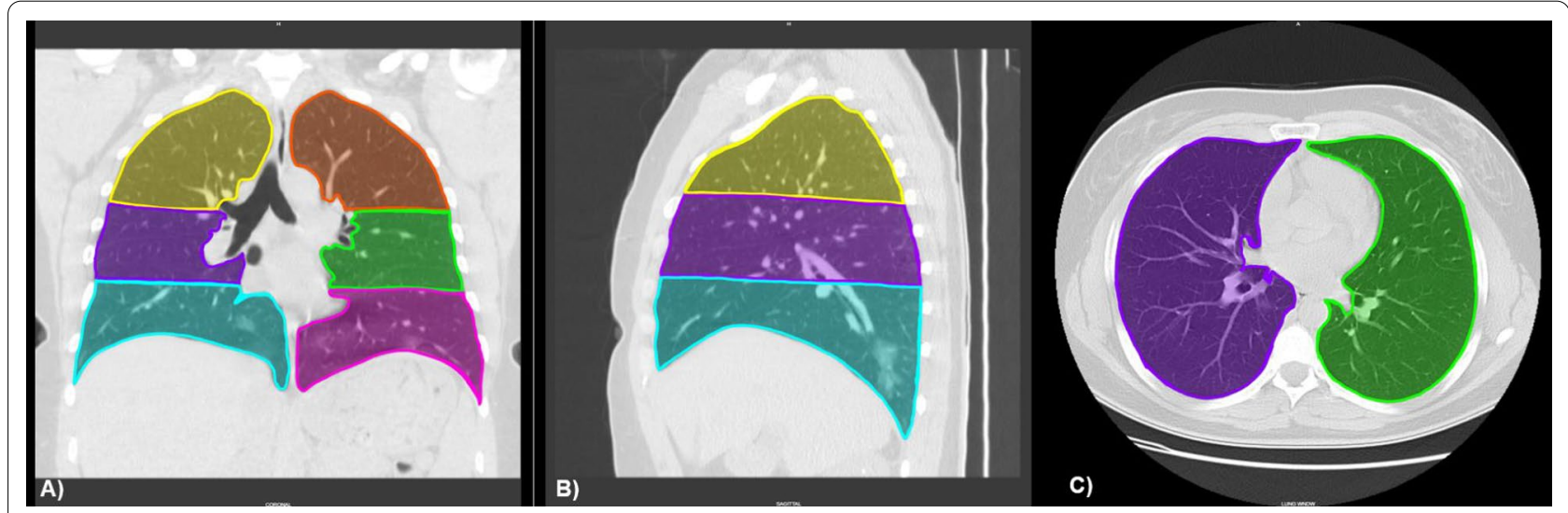

Fig. 1 Ichikado pulmonary zones. The upper zone is defined as the lung parenchyma above the carina, the middle zone as the area between the carina and the pulmonary vein, and the lower zone as the infrapulmonary vein area. A Coronal lung window of CT chest showing all 6 Ichikado pulmonary zones. B Sagittal lung window of CT chest showing right lung Ichikado areas. C Axial lung window of CT chest showing right and left middle zones. This example shows images of a 34-year-old lady with coronavirus disease 2019 


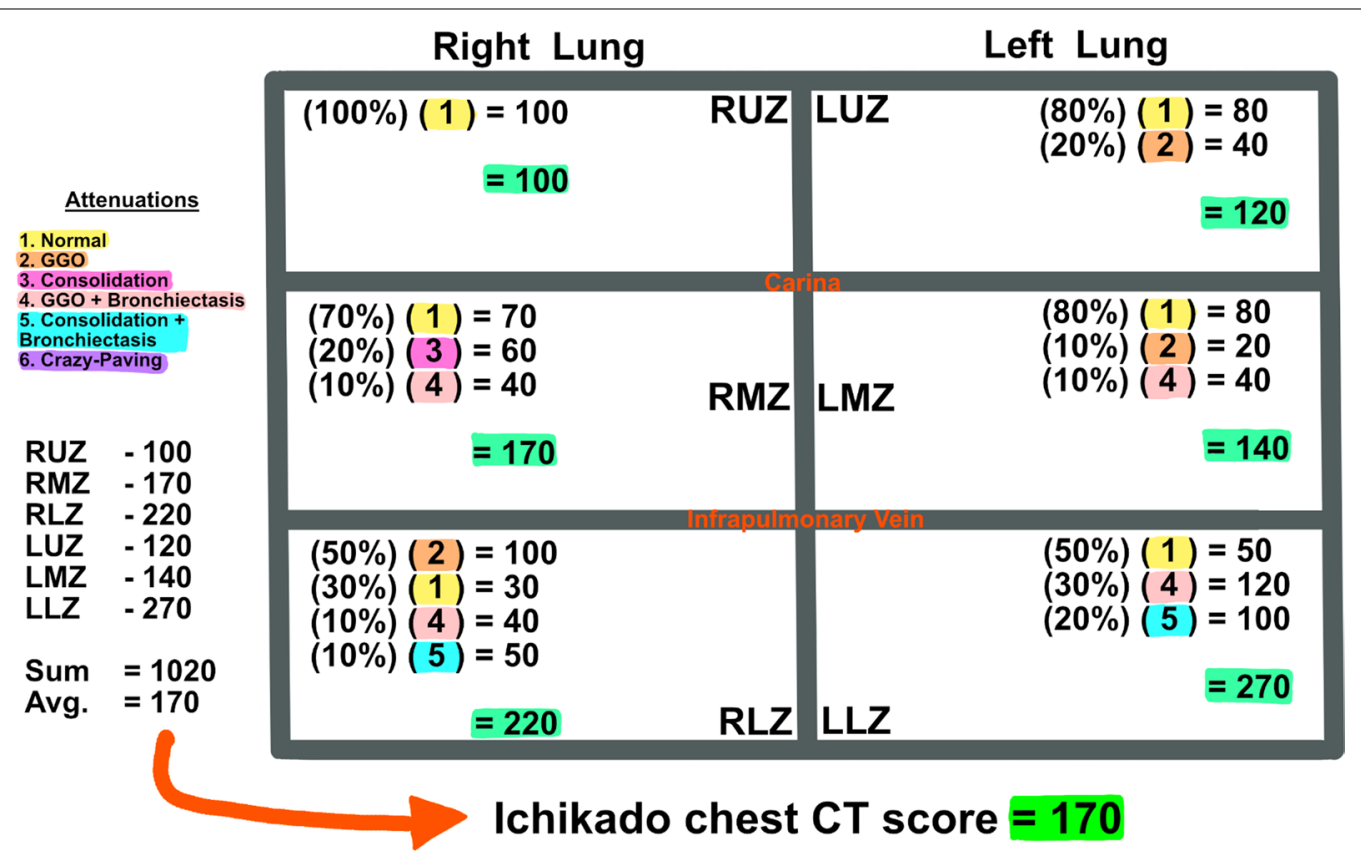

Fig. 2 Example of Ichikado chest CT score calculation. CT Computed tomography, RUZ right upper zone, RMZ right middle zone, RLZ right lower zone, LUZ left upper zone, LMZ left middle zone, LLZ left lower zone, GGO ground-glass opacification

in multivariable approach were those that have been reported with higher risk of adverse events; to avoid overfitting, we excluded factors for which $P>0.1$ in univariable analysis, as well as those that significantly correlated with Ichikado CT score in Spearman's analysis.

The Kaplan-Meier method was utilized to evaluate the relationship between $\mathrm{CT}$ score and all-cause mortality. Receiver operator characteristic (ROC) curve analysis was used to determine the optimal cutoff value of Ichikado $\mathrm{CT}$ score that yielded the highest sensitivity and specificity. The area under the ROC curve (AUC) was used to assess the performance of the discrimination models based on Ichikado CT score.

Statistical analysis was performed based on nonmissing data using IBM SPSS, version 26.0, and missing data were not imputed. All tests were two-sided and a $P$-value $<0.05$ was considered statistically significant. The authors assume responsibility for the accuracy and completeness of the data and analysis, as well as the fidelity of the study.

\section{Results}

A total of 241 patients were admitted at our institution's COVID-19 units, of which 235 were included in our analysis. Five patients were excluded due to missing chest CT scan within $24 \mathrm{~h}$ of admission, and one patient due to missing demographic or clinical data; none of the patients excluded died during hospitalization (Fig. 3). Demographic characteristics and clinical variables on the first $24 \mathrm{~h}$ from hospitalization for all 235 patients are reported in Table 1.

One hundred twenty-nine $(54.9 \%)$ patients were male and $106(45.1 \%)$ were female, with a mean age of $56.0 \pm 15.6$. The most common races were Hispanics (44.2\%) and African Americans (31.1\%), followed by Caucasians (18.3\%). Hypertension (50.3\%) and diabetes mellitus (37.9\%) were the most common comorbidities, followed by COPD (34.0\%), CKD (26.8\%) and ESRD (6.0\%). At the time of CT scan and bloodwork obtention, 110 (46.8\%) patients were on nasal cannula, $81(34.5 \%)$ on high-flow nasal cannula (HFNC), and 8 (3.4\%) were endotracheally intubated. During hospitalization, 59 (25.1\%) developed AKI. Forty-two (17.9\%) patients had new requirements for invasive mechanical ventilation. One hundred ninety-seven $(83.8 \%)$ patients survived to hospital discharge, while $38(16.2 \%)$ died during their hospital stay. When compared to nonsurvivors, survivors were younger $(P<0.001)$, more likely to be women $(P=0.011)$ and had less days since onset of symptoms until hospitalization $(P=0.003)$. The median Ichikado CT score for nonsurvivors was 215.8 (179.6240.0 ), which was significantly higher compared to survivors who had a median score of 138.3 (119.2-165.8) $(P<0.001)$. The interobserver variability among radiologists in calculating Ichikado CT score was excellent 


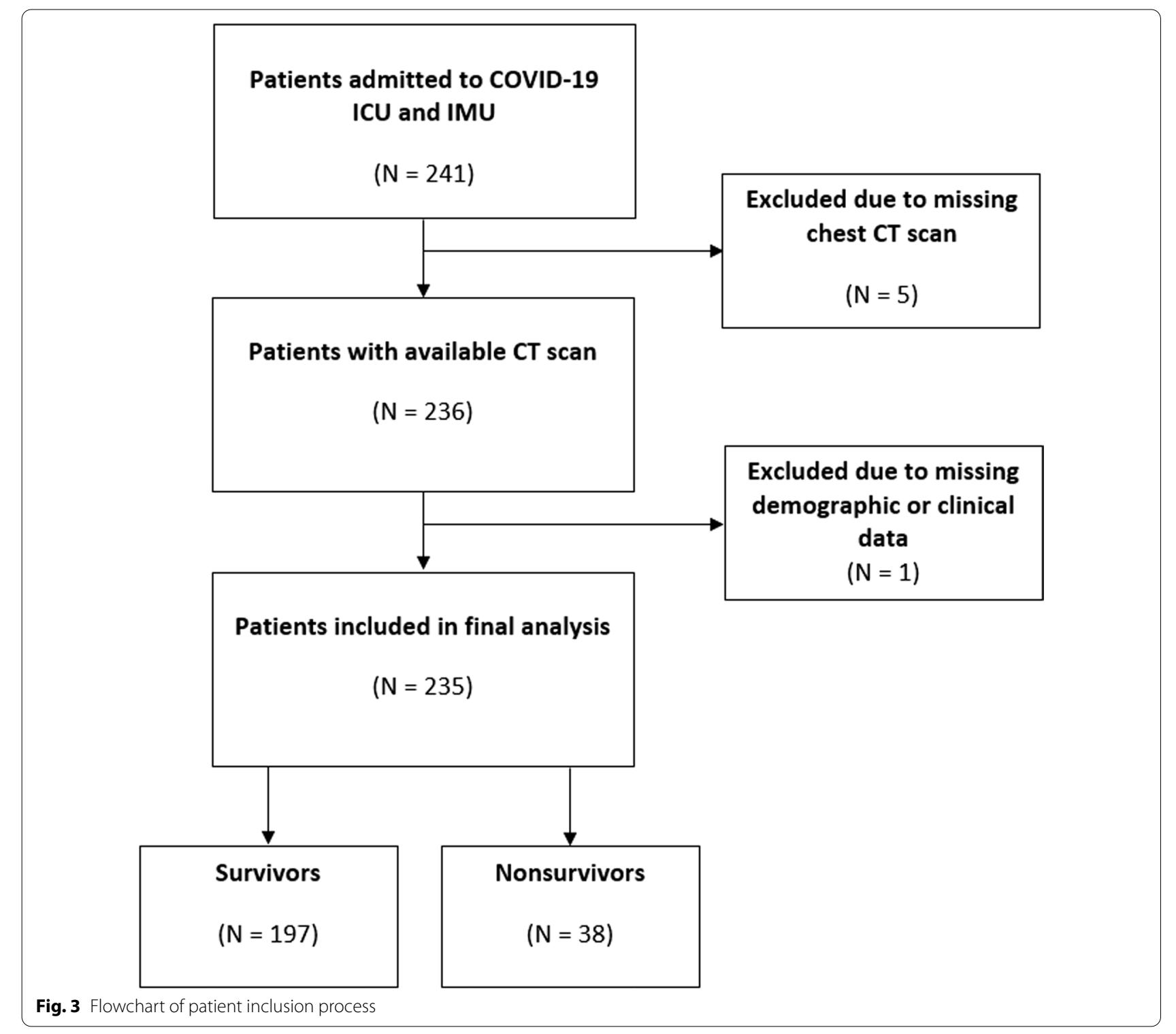

with an intraclass correlation coefficient of 0.992 (95\% CI, 0.989-0.994; $P<0.001$ ).

Analysis by Spearman's correlation coefficient $(R)$ indicated that Ichikado $C T$ score was significantly associated with age $(R=0.243 ; P<0.001)$, days from onset of symptoms to hospitalization $(R=0.270 ; P<0.001)$, hospital length of stay $(R=0.414 ; P<0.001), \mathrm{FiO}_{2}(R=0.425$; $P<0.001), \mathrm{PaO}_{2}: \mathrm{FiO}_{2}(R=-0.530 ; P<0.001), \mathrm{A}$-a gradient $(R=0.481 ; P<0.001)$, SOFA score $(R=0.260 ; P<0.001)$, absolute neutrophil count $(R=0.361 ; P<0.001)$, absolute lymphocyte count $(R=-0.366 ; P<0.001)$ and NLR $(R=0.493 ; P<0.001)$.

Receiver operator characteristic curve analysis yielded an optimal cutoff value of an Ichikado CT score of 172 for prediction of mortality, with a sensitivity of $84.2 \%$ and a specificity of $79.7 \%$ (AUC $=0.873$ ); optimal cutoff value of CT score for prediction of new requirement of invasive mechanical ventilation was 170 , with a sensitivity of $82.4 \%$ and a specificity of $79.3 \%$ (AUC $=0.870$ ) (Fig. 4). A Kaplan-Meier survival curve was performed utilizing the ROC curve cutoff value for Ichikado CT score. By this method, a CT score $\geq 172$ was predictive of all-cause hospital mortality (Fig. 5).

Table 2 summarizes univariable and multivariable Cox regression analysis results for all-cause mortality. On univariable analysis, an Ichikado CT score $\geq 172$ was a statistically significant predictor for mortality with a hazard ratio (HR) of 6.864 (95\% CI, 2.843-16.572; $P<0.001$ ). 
Table 1 Demographic and clinical characteristics of patients

\begin{tabular}{|c|c|c|c|c|}
\hline Characteristic & All $(N=235)$ & Survivors $(N=197)$ & Nonsurvivors $(N=38)$ & $P$-value \\
\hline Age $(y)^{\dagger}$ & $56.0 \pm 15.6$ & $53.9 \pm 15.2$ & $67.1 \pm 13.1$ & 0.000 \\
\hline Gender & & & & 0.011 \\
\hline Male $\boldsymbol{\nabla}$ & $129(54.9 \%)$ & $101(51.3 \%)$ & $28(73.7 \%)$ & \\
\hline Female $\boldsymbol{\nabla}$ & $106(45.1 \%)$ & $96(48.7 \%)$ & $10(26.3 \%)$ & \\
\hline Race & & & & 0.804 \\
\hline African-American $\boldsymbol{\nabla}$ & $73(31.1 \%)$ & $60(30.5 \%)$ & $13(34.2 \%)$ & \\
\hline Caucasian $\boldsymbol{\nabla}$ & $43(18.3 \%)$ & $38(19.3 \%)$ & $5(13.1 \%)$ & \\
\hline Hispanic $\boldsymbol{\nabla}$ & $104(44.2 \%)$ & $86(43.6 \%)$ & $18(47.4 \%)$ & \\
\hline Other $\boldsymbol{\nabla}$ & $15(6.4 \%)$ & $13(6.6 \%)$ & $2(5.3 \%)$ & \\
\hline \multicolumn{5}{|l|}{ Comorbidities } \\
\hline Hypertension $\boldsymbol{\nabla}$ & $119(50.3 \%)$ & 85 (43.1\%) & 34 (89.5\%) & 0.000 \\
\hline Diabetes mellitus $\mathbf{\nabla}$ & $89(37.9 \%)$ & $62(31.5 \%)$ & $27(71.1 \%)$ & 0.000 \\
\hline COPD $\boldsymbol{\nabla}$ & $80(34.0 \%)$ & $56(28.4 \%)$ & $24(63.2 \%)$ & 0.000 \\
\hline$C K D \nabla$ & $63(26.8 \%)$ & $38(19.3 \%)$ & $25(65.8 \%)$ & 0.000 \\
\hline ESRD $\boldsymbol{\nabla}$ & $14(6.0 \%)$ & $11(5.6 \%)$ & $3(7.9 \%)$ & 0.582 \\
\hline $\mathrm{PaO}_{2}{\text { (Torr })^{\ddagger}}$ & $76.0(64.0-88.0)$ & $77.0(65.0-88.0)$ & $70.5(62.2-86.0)$ & 0.216 \\
\hline $\mathrm{PaCO}_{2}{\text { (Torr })^{\ddagger}}^{\ddagger}$ & $38.0(35.0-41.0)$ & $38.0(35.0-41.0)$ & $35.5(33.0-40.2)$ & 0.131 \\
\hline $\mathrm{FiO}_{2}(\%)^{\ddagger}$ & $33.0(28.0-40.0)$ & $32.0(28.0-40.0)$ & $50.0(40.0-100.0)$ & 0.000 \\
\hline $\mathrm{PaO}_{2}: \mathrm{FiO}_{2}{ }^{\neq}$ & $217.8(157.5-275.0)$ & $230.3(171.1-285.7)$ & $140.1(70.2-215.6)$ & 0.000 \\
\hline Mild $(\geq 200) \nabla$ & $132(56.2 \%)$ & $121(61.4 \%)$ & $11(29.0 \%)$ & \\
\hline Moderate $(100-200) \boldsymbol{\nabla}$ & $67(28.5 \%)$ & $57(28.9 \%)$ & $10(26.3 \%)$ & \\
\hline Severe $(<100) \boldsymbol{\nabla}$ & $36(15.3 \%)$ & $19(9.7 \%)$ & $17(44.7 \%)$ & \\
\hline Oxygen delivery device & & & & 0.000 \\
\hline Room air $\boldsymbol{\nabla}$ & $19(8.1 \%)$ & $19(9.6 \%)$ & $0(0 \%)$ & \\
\hline Nasal cannula $\boldsymbol{\nabla}$ & $110(46.8 \%)$ & $101(51.3 \%)$ & $9(23.7 \%)$ & \\
\hline HFNC $\boldsymbol{\nabla}$ & $81(34.5 \%)$ & $62(31.5 \%)$ & $19(50 \%)$ & \\
\hline $\mathrm{BIPAP/CPAP} \boldsymbol{\nabla}$ & $13(5.5 \%)$ & $9(4.6 \%)$ & $4(10.5 \%)$ & \\
\hline Non-rebreather mask $\boldsymbol{\nabla}$ & $4(1.7 \%)$ & $3(1.5 \%)$ & $1(2.6 \%)$ & \\
\hline Endotracheal tube $\boldsymbol{\nabla}$ & $8(3.4 \%)$ & $3(1.5 \%)$ & $5(13.2 \%)$ & \\
\hline Onset of symptoms to hospitalization (days) & $7.0(5.0-10.0)$ & $7.0(5.0-10.0)$ & $10.0(6.5-14.0)$ & 0.003 \\
\hline Hospital length of stay (days) $)^{\ddagger}$ & $10.0(7.0-15.0)$ & $9.0(6.5-14.0)$ & $12.0(7.0-19.0)$ & 0.045 \\
\hline AKI during hospitalization $\boldsymbol{\nabla}$ & $59(25.1 \%)$ & $27(13.7 \%)$ & $32(84.2 \%)$ & 0.000 \\
\hline Ichikado CT score ${ }^{\ddagger}$ & $146.7(121.7-188.3)$ & $138.3(119.2-165.8)$ & $215.8(179.6-240.0)$ & 0.000 \\
\hline A-a gradient $(\text { Torr })^{\ddagger}$ & $119.7(81.1-181.2)$ & $109.3(73.6-161.1)$ & $235.7(134.3-562.6)$ & 0.000 \\
\hline SOFA score ${ }^{\ddagger}$ & $3.0(2.0-4.0)$ & $3.0(2.0-4.0)$ & $4.0(3.0-5.0)$ & 0.000 \\
\hline Absolute neutrophil count $\times 10^{9} / \mathrm{L}^{\ddagger}$ & $4.8(3.4-7.4)$ & $4.7(3.3-6.5)$ & $6.7(3.6-10.7)$ & 0.005 \\
\hline Absolute lymphocyte count $\times 10^{9} / \mathrm{L}^{\ddagger}$ & $1.1(0.7-1.7)$ & $1.2(0.8-1.7)$ & $0.7(0.6-1.0)$ & 0.000 \\
\hline $\mathrm{NLR}^{\ddagger}$ & $4.3(2.7-8.3)$ & $3.7(2.5-6.9)$ & $9.4(5.9-13.3)$ & 0.000 \\
\hline \multicolumn{5}{|l|}{ Treatment modalities } \\
\hline Corticosteroids $\boldsymbol{\nabla}$ & $235(100 \%)$ & $197(100 \%)$ & $38(100 \%)$ & - \\
\hline Ascorbic acid $\boldsymbol{\nabla}$ & $235(100 \%)$ & $197(100 \%)$ & $38(100 \%)$ & - \\
\hline Thiamine $\boldsymbol{\nabla}$ & $235(100 \%)$ & $189(95.9 \%)$ & $38(100 \%)$ & - \\
\hline Anticoagulation $\boldsymbol{\nabla}$ & $227(96.6 \%)$ & $7(3.6 \%)$ & $38(100 \%)$ & 0.206 \\
\hline Tocilizumab $\mathbf{\nabla}$ & $17(7.2 \%)$ & $7(3.6 \%)$ & $10(26.3 \%)$ & 0.000 \\
\hline Remdesivir $\boldsymbol{\nabla}$ & $10(4.3 \%)$ & 197 (100\%) & $3(7.9 \%)$ & 0.225 \\
\hline Prone positioning $\boldsymbol{\nabla}$ & $142(60.4 \%)$ & $109(55.3 \%)$ & $33(86.8 \%)$ & 0.000 \\
\hline $\mathrm{RRT} \boldsymbol{\nabla}$ & $31(13.2 \%)$ & $24(12.2 \%)$ & $7(18.4 \%)$ & 0.298 \\
\hline Vasopressor use $\boldsymbol{\nabla}$ & $53(22.6 \%)$ & $17(8.6 \%)$ & $36(94.7 \%)$ & 0.000 \\
\hline
\end{tabular}

For continuous variables, comparison of groups (survivors and nonsurvivors) was determined using the Student's $t$ test or Mann-Whitney $\mathrm{U}$ test, as appropriate. Chisquared test was utilized for categorical variables. Statistically significant values $(P<0.05)$ are in bold. $A$-a gradient alveolar-arterial gradient, $N L R$ absolute neutrophilto-lymphocyte ratio, $\mathrm{AKl}$ acute kidney injury, $\mathrm{PaCO}_{2}$ arterial partial pressure of carbon dioxide, $\mathrm{PaO}_{2}$ arterial partial pressure of oxygen, $\mathrm{PaO}_{2}: \mathrm{FiO}_{2}$ arterial partial 
Table 1 (continued)

pressure of oxygen-to-fraction of inspired oxygen ratio, CPAP BiPAP bilevel positive airway pressure, CKD chronic kidney disease, COPD chronic obstructive pulmonary disease, continuous positive airway pressure, ESRD end-stage renal disease, fio ${ }_{2}$ fraction of inspired oxygen, $H F N C$ high-flow nasal cannula, $R R T$ renal replacement therapy, SOFA score Sequential Organ Failure Assessment Score

${ }^{\dagger}$ Data are mean \pm standard deviation

* Data are median (interquartile range)

$\checkmark$ Data are frequency (\%)
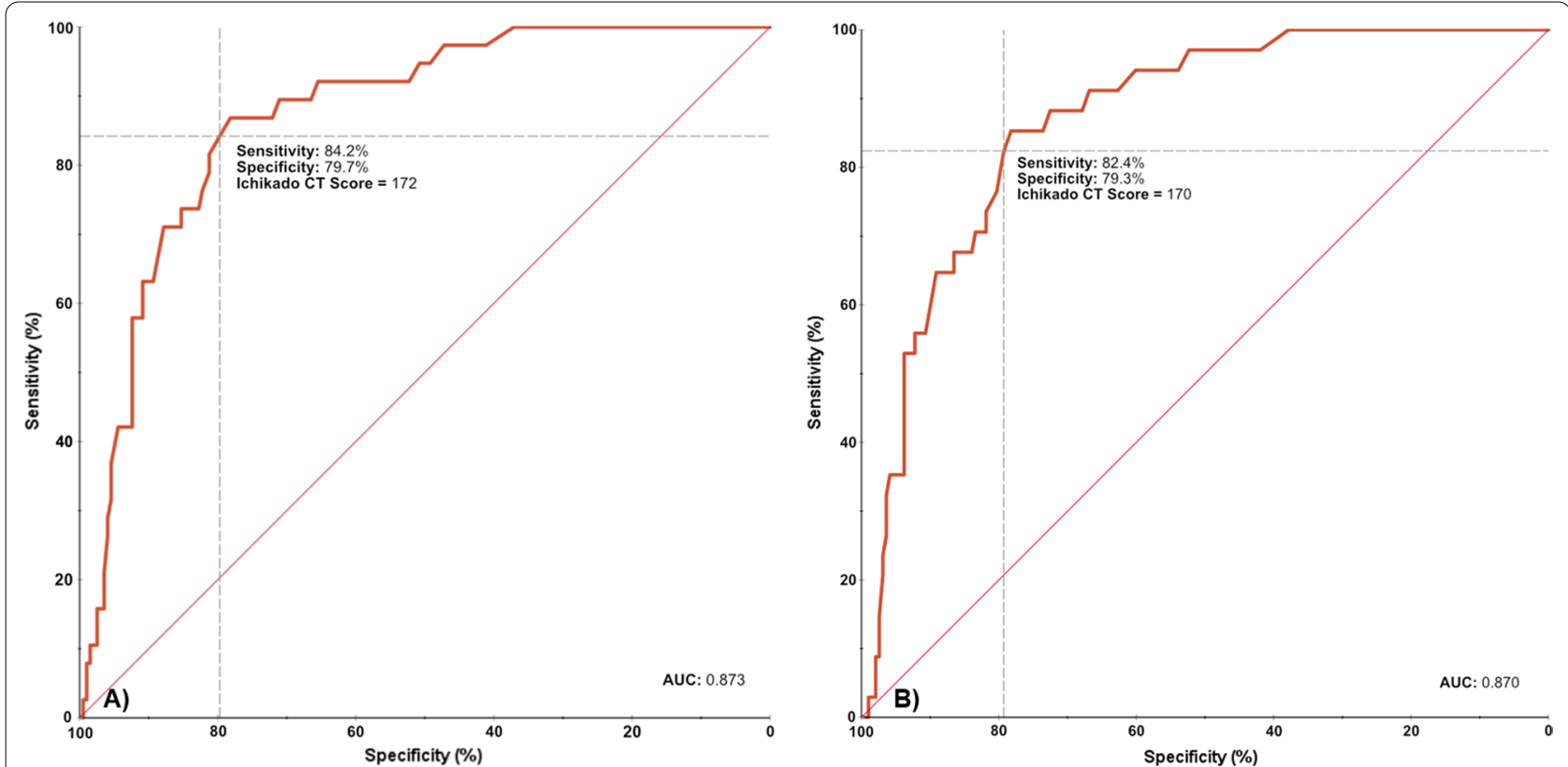

Fig. 4 Receiver operator characteristic curves for cutoff values of CT scores with highest sensitivities and specificities. A Ichikado CT score of 172 has an $84.2 \%$ sensitivity and $79.7 \%$ specificity for predicting all-cause mortality; area under the curve (AUC) $=0.873$. B lchikado CT score of 170 has an $82.4 \%$ sensitivity and $79.3 \%$ specificity for predicting new requirement of invasive mechanical ventilation; $A \cup C=0.870$

Other significant variables for prediction of mortality were age (HR, 1.033; 95\% CI, 1.010-1.058; $P=0.005)$, hypertension (HR, 4.621; 95\% CI, 1.627-13.121; $P=0.004$ ), diabetes mellitus (HR, 2.533; 95\% CI, 1.248 5.139; $P=0.010)$, COPD (HR, 2.205; 95\% CI, 1.1354.285; $P=0.020$ ), CKD (HR, 3.348; 95\% CI, 1.698-6.602; $P<0.001)$, days from onset of symptoms to hospitalization (HR, 1.056; 95\% CI, 1.005-1.109; $P=0.032$ ), AKI during hospitalization (HR, 9.169; 95\% CI, 3.806-22.085; $P<0.001$ ), A-a gradient (HR, 1.003; 95\% CI, 1.001-1.004; $P<0.001$ ), SOFA score (HR, 1.189; 95\% CI, 1.054-1.341; $P=0.005)$, absolute neutrophil count (HR, 1.123; 95\% CI, 1.060-1.190; $P<0.001$ ), absolute lymphocyte count (HR, 0.442; 95\% CI, 0.220-0.891; $P=0.022$ ), NLR (HR, 1.039; 95\% CI, 1.021-1.058; $P<0.001)$, vasopressor use during hospitalization (HR, 33.935; 95\% CI, 8.113-141.942; $P<0.001$ ).

Multivariate analysis showed that Ichikado CT score (HR, 7.772; 95\% CI, 3.164-19.095; $P<0.001$ ), together with age (HR, 1.030; 95\% CI, 1.030-1.060; $P=0.043)$, were independent predictors of all-cause in-hospital mortality. Additionally, an Ichikado CT score $\geq 172$ (HR, 22.480; 95\% CI, 8.161-61.921; $P<0.001$ ) was an independent predictor of new requirement of invasive mechanical ventilation during hospitalization.

\section{Discussion}

Ichikado et al. previously validated a CT score in patients with ARDS, identifying a score above 230 as an independent predictor of mortality [20]. In our single-center retrospective cohort study, we recognized the Ichikado CT score as an independent predictor of both requiring mechanical ventilation and all-cause mortality in patients hospitalized with COVID-19 pneumonia. However, our cutoff value with highest sensitivity and specificity for prediction of mortality was 172 . We believe this difference is explained mainly by the time to obtention of the CT scans. In contrast with their study, which analyzed scans up to 7 days from the onset of ARDS, we only included CT scans taken in the first $24 \mathrm{~h}$ of hospital 


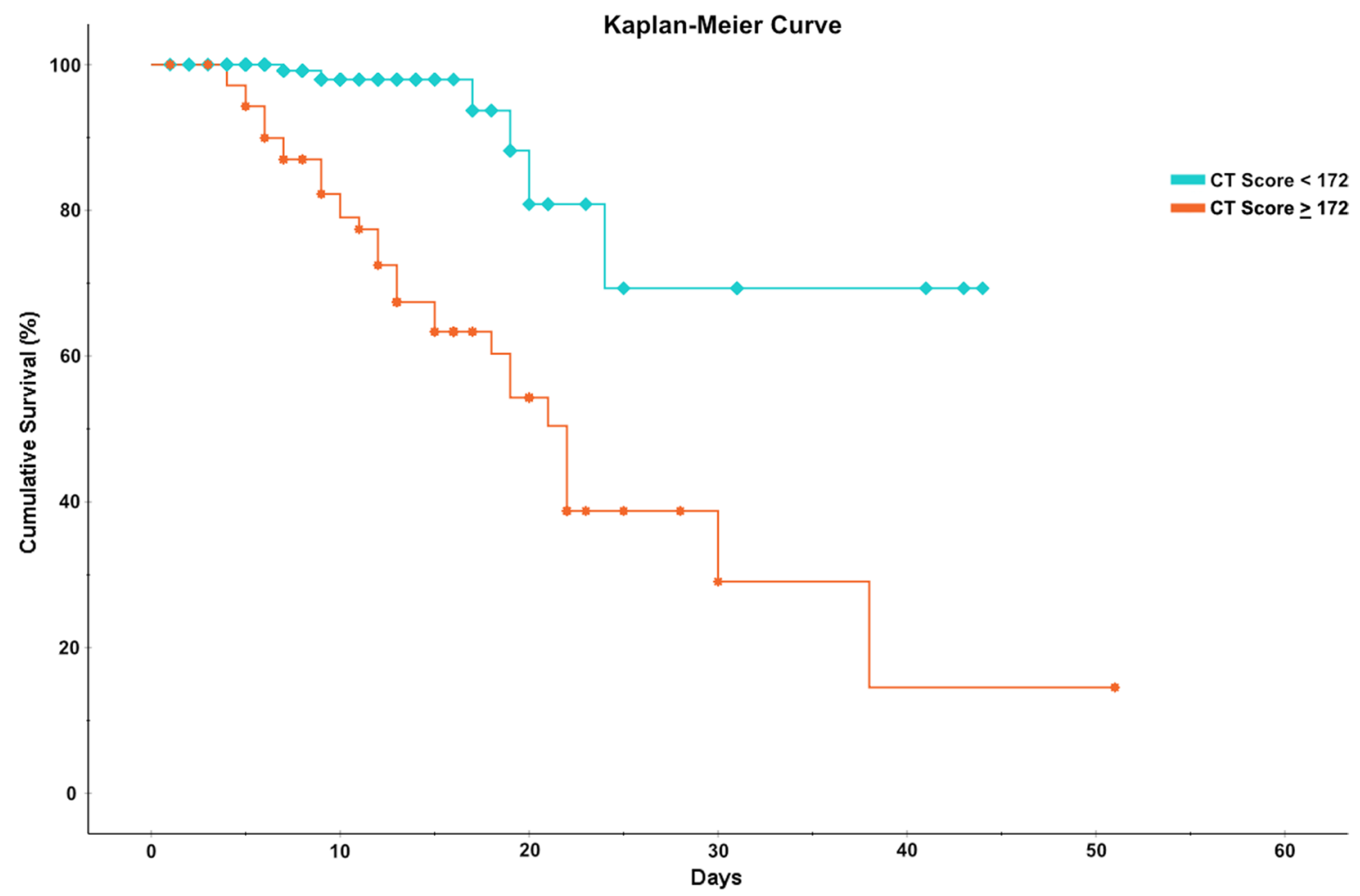

Fig. 5 Kaplan-Meier survival curve. Cumulative survival rate comparison between COVID-19 patients with admission Ichikado CT score $\geq 172$ and $<172$. Percentage of cumulative survival is expressed on the $y$-axis, while time (days) of the observation period is expressed on the $x$-axis. CT Computed tomography

admission. It has previously been described that COVID19 CT imaging features will vary drastically according to the stage of disease. Early on, patients will have predominantly small areas of GGOs, compared to the late phase of disease (12-17 days after onset of symptoms), during which patients will have bilateral consolidations mixed with GGOs, and even crazy-paving pattern [22, 23]. The presence of fibroproliferative attenuations in chest $\mathrm{CT}$ scans obtained in later stages of the disease, could potentially explain the discrepancies between their study and ours. In addition, the CT findings of "typical" ARDS differ considerably from that of COVID-19 organizing pneumonia [24].

Multiple CT scoring models have been proposed in COVID-19 for quantifying severity of disease, and predicting prognosis. Regardless of the CT scoring system analyzed, all prior studies have identified a CT score as an independent predictor of adverse outcome in patients with COVID-19 pneumonia [10, 15, 17, 18]. Moreover, $\mathrm{CT}$ scores had significant associations with various inflammatory biomarkers known to be predictors of mortality $[14,16]$. However, to our knowledge, no other study has followed the Ichikado scoring method, which we believe might be superior to previously described methods due to its reproducibility and having both a quantitative and qualitative approach.

Our multivariate regression results showed that an Ichikado CT score $\geq 172$ was the highest independent predictor of all-cause in-hospital mortality. Furthermore, a higher absolute lymphocyte count at the time of hospital admission was associated with significantly decreased odds of mortality. These findings are consistent with prior reports [25-27].

The role of chest CT scan in the diagnosis and management of COVID-19 remains controversial. While some authors and radiological societies recommend against routine use of CT scan in patients with COVID-19, our analysis seems to suggest a benefit by allowing early recognition of patients at high risk of decompensation [28, 29]. Additionally, the high diagnostic sensitivity may prove to be an asset as CT scans may show characteristic findings of the disease even when RT-PCR is negative (false-negative), or in hospitals with a relatively long turn-around time for RT-PCR results [16, 30].

There were limitations in our analysis. Due to the single-center retrospective observational nature of the study, we cannot exclude the possibility of unmeasured confounders. Furthermore, despite including laboratory 
Table 2 Univariable and multivariable Cox regression analysis to predict all-cause mortality

\begin{tabular}{|c|c|c|c|c|c|c|}
\hline \multirow[b]{2}{*}{ Variable } & \multicolumn{3}{|c|}{ Univariable analysis } & \multicolumn{3}{|c|}{ Multivariable analysis } \\
\hline & Hazard ratio & $\mathbf{9 5 \%}$ confidence interval & $P$-value & Hazard ratio & 95\% confidence interval & $P$-value \\
\hline Age (y) & 1.033 & $1.010-1.058$ & 0.005 & 1.030 & $1.001-1.060$ & 0.043 \\
\hline \multicolumn{7}{|l|}{ Gender } \\
\hline Male & 1.425 & $0.682-2.979$ & 0.346 & - & - & - \\
\hline Female & 1 (ref) & & & & & \\
\hline Race & & & 0.862 & - & - & - \\
\hline African-American & 1 (ref) & & & & & \\
\hline Caucasian & 0.652 & $0.232-1.832$ & 0.417 & & & \\
\hline Hispanic & 0.808 & $0.394-1.654$ & 0.559 & & & \\
\hline Other & 0.916 & $0.206-4.072$ & 0.908 & - & - & - \\
\hline \multicolumn{7}{|l|}{ Comorbidities } \\
\hline Hypertension & 4.621 & $1.627-13.121$ & 0.004 & 2.056 & $0.665-6.359$ & 0.211 \\
\hline Diabetes mellitus & 2.533 & $1.248-5.139$ & 0.010 & 2.004 & $0.940-4.273$ & 0.072 \\
\hline COPD & 2.205 & $1.135-4.285$ & 0.020 & - & - & - \\
\hline CKD & 3.348 & $1.698-6.602$ & 0.000 & - & - & - \\
\hline ESRD & 0.788 & $0.241-2.574$ & 0.693 & - & - & - \\
\hline $\mathrm{PaO}_{2}$ (Torr) & 0.991 & $0.975-1.007$ & 0.284 & - & - & - \\
\hline $\mathrm{PaCO}_{2}$ (Torr) & 1.005 & $0.971-1.040$ & 0.789 & - & - & - \\
\hline $\mathrm{FiO}_{2}(\%)$ & 1.020 & $1.009-1.031$ & 0.000 & - & - & - \\
\hline $\mathrm{PaO}_{2}: \mathrm{FiO}_{2}$ & 0.993 & $0.988-0.997$ & 0.001 & - & - & - \\
\hline Oxygen delivery device & & & 0.085 & - & - & - \\
\hline Room air & - & - & - & & & \\
\hline Nasal cannula & 0.194 & $0.064-0.587$ & 0.004 & & & \\
\hline HFNC & 0.459 & $0.169-1.242$ & 0.125 & & & \\
\hline $\mathrm{BiPaP/CPAP}$ & 0.383 & $0.102-1.441$ & 0.156 & & & \\
\hline Non-rebreather mask & 0.882 & $0.101-7.730$ & 0.910 & & & \\
\hline Endotracheal tube & 1 (ref) & & & & & \\
\hline $\begin{array}{l}\text { Onset of symptoms to hospitalization } \\
\text { (days) }\end{array}$ & 1.056 & $1.005-1.109$ & 0.032 & - & - & - \\
\hline AKI during hospitalization & 9.169 & $3.806-22.085$ & 0.000 & - & - & - \\
\hline Ichikado CT score & 1.010 & $1.005-1.014$ & 0.000 & & & \\
\hline$\geq 172$ & 6.864 & $2.843-16.572$ & 0.000 & & 3.164-19.095 & 0.000 \\
\hline$<172$ & 1 (ref) & & & 7.772 & & \\
\hline A-a gradient (Torr) & 1.003 & $1.001-1.004$ & 0.000 & - & - & - \\
\hline SOFA score & 1.189 & $1.054-1.341$ & 0.005 & - & - & - \\
\hline Absolute neutrophil count $\times 10^{9} / \mathrm{L}$ & 1.123 & $1.060-1.190$ & 0.000 & - & - & - \\
\hline Absolute lymphocyte count $\times 10^{9} / \mathrm{L}$ & 0.442 & $0.220-0.891$ & 0.022 & - & - & - \\
\hline NLR & 1.039 & $1.021-1.058$ & 0.000 & - & - & - \\
\hline \multicolumn{7}{|l|}{ Treatment modalities } \\
\hline Steroids & - & - & - & - & - & - \\
\hline Ascorbic acid & - & - & - & - & - & - \\
\hline Thiamine & - & - & - & - & - & - \\
\hline Anticoagulation & - & - & - & - & - & - \\
\hline Tocilizumab & 1.743 & $0.822-3.693$ & 0.147 & - & - & - \\
\hline Remdesivir & 1.162 & $0.354-3.811$ & 0.805 & - & - & - \\
\hline Prone positioning & 1.674 & $0.643-4.359$ & 0.291 & - & - & - \\
\hline RRT & 0.812 & $0.354-1.860$ & 0.622 & - & - & - \\
\hline Vasopressor use & 33.935 & $8.113-141.942$ & 0.000 & - & - & - \\
\hline
\end{tabular}


data obtained on the same day as the chest CT, we did not provide correlation analysis with biomarkers (e.g., interleukin-6, C-reactive protein, D-dimer, ferritin, etc.) known to be predictors of mortality in COVID-19. External validation of our analysis is required as most of the studied patients, unless medically contraindicated, received the MATH + protocol (methylprednisolone, IV ascorbic acid, thiamine, heparin, etc.) which is not the standard of care in most institutions, and could potentially be a confounder in our study [31,32].

\section{Conclusions}

Further prospective evaluation of the Ichikado CT score in patients with COVID-19 will be necessary not only to clarify its prognostic value, but also to assess its potential application in stratifying severity of disease, guiding treatment, and monitoring disease progression. Ideally, such investigations would help identify the patients at high risk of deterioration who require prompt initiation of treatment and ICU monitoring.

In summary, this study provided evidence that Ichikado CT score obtained in the first $24 \mathrm{~h}$ of hospital admission, is an independent predictor of requiring invasive mechanical ventilation and all-cause in-hospital mortality in patients with COVID-19 pneumonia. Since COVID-19 is a potentially fatal disease, utilizing the Ichikado CT score may aid in appropriately triaging patients, so that those with severe disease can timely initiate more aggressive treatment under closer monitoring.

\begin{abstract}
Abbreviations
95\% Cl: 95\% Confidence interval; A-a gradient: Alveolar-arterial gradient; ABG: Arterial blood gas; AKI: Acute kidney injury; ARDS: Acute respiratory distress syndrome; AUC: Area under roc curve; CKD: Chronic kidney disease; COPD: Chronic obstructive pulmonary disease; COVID-19: Coronavirus disease 2019; $\mathrm{CT}$ : Computed tomography; ESRD: End-stage renal disease; $\mathrm{FiO}_{2}$ : Fraction of inspired oxygen; GGO: Ground-glass opacity; HFNC: High-flow nasal cannula; HR: Hazard ratio; ICU: Intensive care unit; IMU: Intermediate care unit; IQR: Interquartile range; LoS: Length of stay; MATH + protocol: Methylprednisolone, IV ascorbic acid, thiamine, heparin, etc.; NLR: Absolute neutrophil-to-lymphocyte ratio; $\mathrm{PaCO}_{2}$ : Partial pressure of arterial carbon dioxide; $\mathrm{PaO}_{2}$ : Partial pressure of arterial oxygen; $\mathrm{PaO}_{2} \mathrm{FiO}_{2}$ : Arterial partial pressure of oxygen-tofraction of inspired oxygen ratio; $R$ : Spearman's correlation coefficient; RRT: Renal replacement therapy; ROC curve: Receiver operator characteristic curve; RT-PCR: Reverse transcription polymerase chain reaction; SARS-CoV-2: Severe acute respiratory syndrome coronavirus 2; SD: Standard deviation; SOFA Score: Sequential Organ Failure Assessment Score; SOFIA: SARS antigen fluorescent immunoassay.
\end{abstract}

\section{Acknowledgements}

Not applicable.

\section{Guarantor}

The authors assume responsibility for the accuracy and completeness of the data and analysis, as well as the fidelity of the study.

\section{Authors' contributions}

All authors were responsible for the study design, study data integrity, data analysis, data interpretation, and preparation of the manuscript. AA, MD and $\mathrm{CP}$ were responsible for data acquisition. $\mathrm{AA}, \mathrm{CP}$ and PM did the statistical analysis. All authors reviewed the manuscript and approved the final submitted version. All authors read and approved the final manuscript.

\section{Funding}

This is a self-funded research; the authors received no financial support for the research, authorship, and/or publication of this article.

\section{Availability of data and materials}

The datasets used and analyzed during the current study are available from the corresponding author on reasonable request.

\section{Declarations}

\section{Ethics approval and consent to participate}

The WCG institutional review board approved this retrospective study and patient consent was waived due to its non-interventional nature (Identifier: 20210799).

\section{Consent for publication \\ Not applicable.}

\section{Competing interests}

The authors declare that they have no competing interests.

\section{Author details}

${ }^{1}$ United Memorial Medical Center, Houston, TX, USA. ${ }^{2}$ Universidad Autónoma de Baja California, Tijuana, México. ${ }^{3}$ Universidad Xochicalco, Ensenada, México. ${ }^{4}$ Benemérita Universidad Autónoma de Puebla, Puebla, México. ${ }^{5}$ University of Texas Health Science Center at Houston, Houston, TX, USA.

Received: 19 May 2021 Accepted: 8 Auqust 2021

Published online: 21 August 2021

\section{References}

1. Wu Z, McGoogan JM. Characteristics of and important lessons from the coronavirus disease 2019 (COVID-19) outbreak in China: summary of a report of 72314 cases from the chinese center for disease control and prevention. JAMA. 2020;323(13):1239-42.

2. Zhu N, Zhang D, Wang W, Li X, Yang B, Song J, et al. A novel coronavirus from patients with pneumonia in China, 2019. New Engl J Med. 2020;382(8):727-33.

3. Mahase E. Covid-19: WHO declares pandemic because of "alarming levels" of spread, severity, and inaction. BMJ. 2020;368:m1036.

4. World Health Organization. COVID-19 Weekly Epidemiological Update 2021. February 2021. https://www.who.int/publications/m/item/weeklyepidemiological-update---16-february-2021. Accessed 17 Feb 2020.

5. Huang C, Wang Y, Li X, Ren L, Zhao J, Hu Y, et al. Clinical features of patients infected with 2019 novel coronavirus in Wuhan, China. Lancet. 2020;395(10223):497-506.

6. Wang D, Hu B, Hu C, Zhu F, Liu X, Zhang J, et al. Clinical characteristics of 138 hospitalized patients with 2019 novel coronavirus-infected pneumonia in Wuhan, China. JAMA. 2020;323(11):1061-9.

7. Tay MZ, Poh CM, Renia L, MacAry PA, Ng LFP. The trinity of COVID19: immunity, inflammation and intervention. Nat Rev Immunol. 2020:20(6):363-74.

8. Berlin DA, Gulick RM, Martinez FJ. Severe Covid-19. New Engl J Med. 2020;383(25):2451-60.

9. Robba C, Battaglini D, Pelosi P, Rocco PRM. Multiple organ dysfunction in SARS-CoV-2: MODS-CoV-2. Expert Rev Respir Med. 2020;14(9):865-8.

10. Mahdjoub E, Mohammad W, Lefevre T, Debray MP, Khalil A. Admission chest CT score predicts 5-day outcome in patients with COVID-19. Intensive Care Med. 2020:46(8):1648-50.

11. Malik P, Patel U, Mehta D, Patel N, Kelkar R, Akrmah M, et al. Biomarkers and outcomes of COVID-19 hospitalisations: systematic review and meta-analysis. BMJ Evid Based Med. 2020. https://doi.org/10.1136/ bmjebm-2020-111536.

12. Kermali M, Khalsa RK, Pillai K, Ismail Z, Harky A. The role of biomarkers in diagnosis of COVID-19—a systematic review. Life Sci. 2020;254:117788. 
13. Tian W, Jiang W, Yao J, Nicholson CJ, Li RH, Sigurslid HH, et al. Predictors of mortality in hospitalized COVID-19 patients: a systematic review and meta-analysis. J Med Virol. 2020;92(10):1875-83.

14. Zhang J, Meng G, Li W, Shi B, Dong H, Su Z, et al. Relationship of chest CT score with clinical characteristics of 108 patients hospitalized with COVID-19 in Wuhan, China. Respir Res. 2020;21(1):180.

15. Xiao J, Li X, Xie Y, Huang Z, Ding Y, Zhao S, et al. Maximum chest CT score is associated with progression to severe illness in patients with COVID-19: a retrospective study from Wuhan, China. BMC Infect Dis. 2020;20(1):953.

16. Francone M, lafrate F, Masci GM, Coco S, Cilia F, Manganaro L, et al. Chest CT score in COVID-19 patients: correlation with disease severity and short-term prognosis. Eur Radiol. 2020. https://doi.org/10.1007/ s00330-020-07033-y.

17. Colombi D, Bodini FC, Petrini M, Maffi G, Morelli N, Milanese G, et al. Well-aerated lung on admitting chest $C T$ to predict adverse outcome in COVID-19 pneumonia. Radiology. 2020;296(2):E86-e96.

18. Yang R, Li X, Liu H, Zhen Y, Zhang X, Xiong Q, et al. Chest CT severity score: an imaging tool for assessing severe COVID-19. Radiol Cardiothorac Imaging. 2020;2(2):e200047.

19. Malpani Dhoot N, Goenka U, Ghosh S, Jajodia S, Chand R, Majumdar S, et al. Assigning computed tomography involvement score in COVID-19 patients: prognosis prediction and impact on management. BJR Open. 2020;2(1):20200024

20. Ichikado K, Suga M, Muranaka H, Gushima Y, Miyakawa H, Tsubamoto M, et al. Prediction of prognosis for acute respiratory distress syndrome with thin-section CT: validation in 44 cases. Radiology. 2006;238(1):321-9.

21. The National Heart, Lung, and Blood Institute ARDS Clinical Trials Network. Higher versus Lower Positive End-Expiratory Pressures in Patients with the Acute Respiratory Distress Syndrome. New Engl I Med. 2004;351(4):327-36

22. Hochhegger B, Zanon M, Altmayer S, Mandelli NS, Stuker G, Mohammed TL, et al. COVID-19 mimics on chest CT: a pictorial review and radiologic guide. Br J Radiol. 2021:94(1118):20200703.

23. Salehi S, Abedi A, Balakrishnan S, Gholamrezanezhad A. Coronavirus disease 2019 (COVID-19): a systematic review of imaging findings in 919 patients. AJR Am J Roentgenol. 2020;215(1):87-93.
24. Kory P, Kanne JP. SARS-CoV-2 organising pneumonia: has there been a widespread failure to identify and treat this prevalent condition in COVID-19? BMJ Open Respir Res. 2020. https://doi.org/10.1136/bmjre sp-2020-000724.

25. Wiersinga WJ, Rhodes A, Cheng AC, Peacock SJ, Prescott HC. Pathophysiology, transmission, diagnosis, and treatment of coronavirus disease 2019 (COVID-19): a review. JAMA. 2020;324(8):782-93.

26. Rosenthal N, Cao Z, Gundrum J, Sianis J, Safo S. Risk factors associated with in-hospital mortality in a US national sample of patients with COVID19. JAMA Netw Open. 2020;3(12):e2029058.

27. Wu C, Chen X, Cai Y, Xia J, Zhou X, Xu S, et al. risk factors associated with acute respiratory distress syndrome and death in patients with coronavirus disease 2019 pneumonia in Wuhan, China. JAMA Intern Med. 2020;180(7):934-43.

28. Hope MD, Raptis CA, Shah A, Hammer MM, Henry TS. A role for CT in COVID-19? What data really tell us so far. Lancet. 2020;395(10231):1189-90.

29. American College of Radiology. ACR Recommendations for the use of Chest Radiography and Computed Tomography (CT) for Suspected COVID-19. March 2020 https://www.acr.org/Advocacy-and-Economics/ ACR-Position-Statements/Recommendations-for-Chest-Radiographyand-CT-for-Suspected-COVID19-Infection. Accessed 17 Feb 2020.

30. Ai T, Yang Z, Hou H, Zhan C, Chen C, Lv W, et al. Correlation of chest CT and RT-PCR testing for coronavirus disease 2019 (COVID-19) in China: a report of 1014 cases. Radiology. 2020;296(2):E32-e40.

31. Marik PE, Kory P, Varon J, Iglesias J, Meduri GU. MATH+ protocol for the treatment of SARS-CoV-2 infection: the scientific rationale. Expert Rev Anti Infect Ther. 2021;19(2):129-35.

32. Kory P, Meduri GU, Iglesias J, Varon J, Marik PE. Clinical and scientific rationale for the "MATH+" hospital treatment protocol for COVID-19. $J$ Intensive Care Med. 2021;36(2):135-56.

\section{Publisher's Note}

Springer Nature remains neutral with regard to jurisdictional claims in published maps and institutional affiliations.
Ready to submit your research? Choose BMC and benefit from:

- fast, convenient online submission

- thorough peer review by experienced researchers in your field

- rapid publication on acceptance

- support for research data, including large and complex data types

- gold Open Access which fosters wider collaboration and increased citations

- maximum visibility for your research: over $100 \mathrm{M}$ website views per year

At $B M C$, research is always in progress.

Learn more biomedcentral.com/submissions 